\title{
Asimetrías y aproximaciones \\ a la problemática educativa de jóvenes indígenas de la Sierra Central ecuatoriana*
}

\author{
Asymmetries and Approaches to the Education \\ Problems of the Indigenous Youth from the Central \\ Highlands of Ecuador*
}

\section{Assimetrias e aproximações à problemática educativa de jovens indígenas da Serra Central equatoriana*}

\section{Daniel Llanos Erazo ${ }^{* *}$ Ecuador}

Recibido el 29 de febrero de 2012, aceptado el 21 de septiembre de 2012

* Este artículo es el resultado de la investigación "Jóvenes indígenas y escuela" adscrito al programa de investigación de Juventud Indígena del Centro de Investigaciones de Niñez, Adolescencia y Juventud de la Universidad Politécnica Salesiana del Ecuador, -UPS-. El mencionado programa se encuentra en ejecución desde junio de 2010 y cuenta con el financiamiento de la UPS. del Ecuador.

** Magíster en Política Social de la Infancia y Adolescencia. Pedagogo. Profesor investigador del Centro de Investigaciones de la Niñez, Adolescencia y Juventud, CINAJ, UPS, Ecuador. Investigador del GT CLACSO “Juventud y Prácticas Políticas en América Latina”. Correo electrónico: danielgllanose@ gmail.com 


\section{Resumen}

Objetivo: analizar los cambios y transformaciones que se han producido en las prácticas socio-educativas de jóvenes indígenas de la Sierra Central de Ecuador. Metodología: esta investigación combina procedimientos y enfoques cuantitativos y cualitativos, siendo el último el predominante, por las continuas aproximaciones etnográficas que se realizaron a 104 comunidades de tres provincias de la Sierra Central (Cotopaxi, Tungurahua y Chimborazo). Se aplicaron entrevistas tematizadas a dirigentes, padres de familia, profesores de los centros educativos y jóvenes de las comunidades estudiadas. Resultados: se evidencian las tensiones que se producen en las comunidades a la hora de evaluar y sopesar los logros alcanzados a partir del ingreso del sistema educativo escolarizado a la vida comunal, ya que las transformaciones y asimetrías que se han generado son nuevas y divergentes a las prácticas comunitarias. Conclusiones: el ingreso de los jóvenes comuneros al sistema educativo provoca cambios culturales en las relaciones familiares y comunitarias, prolongando y afianzando las asimetrías de la vida comunitaria.

Palabras Claves: sistema educativo, jóvenes, comunidad indígena.

\section{Abstract}

Objective: to analyze the changes and transformations that have occurred in the socio-educational practices of indigenous youth from the Central Highlands of Ecuador. Methodology: this research includes quantitative and qualitative approaches and methods. The qualitative approach predominates because of the continuous application of ethnographic approaches, which were followed with 104 communities in three provinces of the Central Highlands (Cotopaxi, Tungurahua and Chimborazo). Thematic interviews were conducted with leaders, parents, school teachers and youth of the studied communities. Results: tensions within the communities were revealed at the time of evaluation and consideration of the attained achievements, especially from the admission of the education system to the community life. This was evidenced because the produced transformations and asymmetries are new and divergent compared to those of the community practices. Conclusions: the admission of the community youth to the education system causes cultural changes in family and community relationships, extending and strengthening the asymmetries of the community life.

82 Keywords: Education system, youth, indigenous community. 


\section{Resumo}

Objetivo: analisar os câmbios e transformações que tem se produzido nas praticas socio-educativas de jovens indígenas da Serra Central de Equador. Metodologia: esta pesquisa mistura procedimentos e enfoques quantitativos e qualitativos, sendo o ultimo o predominante, pelas continuas aproximações etnográficas que realisaram se a 104 comunidades de três províncias da Serra Central (Cotopaxi, Tungurahua e Chimborazo). Aplicaram se entrevistas tematizadas a dirigentes, padres de família, professores dos centros educativos e jovens das comunidades estudadas. Resultados: Evidenciam se as tensões que se produzem nas comunidades à hora de avaliar e sopesar os logros alcançados a partir do ingresso do sistema educativo escolarizado à vida comunal, já que as transformações e assimetrias que tem se gerado são novas e divergentes às praticas comunitárias. Conclusões: $\mathrm{O}$ ingresso dos jovens comuneros ao sistema educativo provoca câmbios culturais nas relaciones familiares e comunitárias, prolongando e afiançando as assimetrias da vida comunitária.

Palavras Chaves: sistema educativo, jovens, comunidade indígena. 


\section{Introducción}

"La educación primitiva era un proceso a través del cual se mantenía una continuidad entre padres e hijos... La educación moderna le da una gran importancia a la función de la enseñanza de crear discontinuidades: de convertir al hijo del analfabeto en un individuo que sabe leer y escribir".

Margaret Mead

Los estudios de juventud, generalmente, se han centrado en temas y problemas referidos a jóvenes de contextos urbanos y sus relaciones con distintos ámbitos de la sociedad (Pérez, 2008). El caso ecuatoriano sigue esta tendencia, centrando su interés en estudios relacionados con culturas juveniles, espacios de socialización familiar, escolar y consumo musical en las urbes (Cerbino, 2000, 2004, 2008; Unda, 2010). En el balance, los trabajos investigativos acerca de jóvenes y de la juventud, han dejado de lado la población de sectores rurales y, más aún, a jóvenes indígenas en contextos de ruralidad que, de manera creciente, desarrollan sus prácticas sociales entre lo rural y lo urbano (Unda, 2011).

Es por ello, que el Centro de Investigaciones de la Niñez, Adolescencia y Juventud, -CINAJ- de la Universidad Politécnica Salesiana de Ecuador, ha desarrollado el programa de investigación de "Juventud Indígena" cuyo principal foco de atención es la constitución y producción de los sujetos comunitarios en contextos de ruralidad y urbanidad constante.

La escuela como institución productora de sujetos es uno de los espacios que privilegia el programa de investigación a través de su proyecto investigativo "Jóvenes indígenas y escuela". Una de los interrogantes que surgen de las primeras aproximaciones al objeto de estudio es: ¿qué relación tiene el proceso educativo escolarizado con la transformación comunitaria en la Sierra Central ecuatoriana? Para intentar responder esta pregunta se decidió realizar aproximaciones etnográficas a comunidades indígenas de la zona central andina del Ecuador. Las características fundamentales de dicha zona radican en su trayectoria histórica, a saber, que las provincias de Cotopaxi, Tungurahua y Chimborazo son altamente habitadas por pueblos kichuas ${ }^{1}$. Pueblos que a lo largo de la historia

1 De acuerdo con el último censo de población y vivienda, 2010, en la provincia de Cotopaxi el 22,1 \% de la población se auto identifica como indígena mientras que en la provincia de Tungurahua el 12,4 $\%$ y en la provincia de Chimborazo el $38 \%$. 
han demostrado resistencia y han sido partícipes de diversas intifadas ${ }^{1}$ en busca de mejores condiciones de vida.

Una de las principales demandas que la población indígena ha realizado en los últimos 50 años ha sido el acceso al sistema educativo formal, anhelo que se concretó con la incursión de misiones católicas, cristianas y evangélicas. Por supuesto, que a esto hay que sumarle el último ingreso de las agencias de cooperación y ONG's que se encargaron de montar un enorme proceso de capacitación, sobre todo, en temas de género, derechos y salud. En suma, el proceso educativo también gira alrededor de la agenda de la cooperación que es la encargada de definir temáticas y ámbitos que los comuneros deben conocer y por lo tanto, son susceptibles de aprendizaje a través, preferencialmente, de talleres comunitarios.

\section{Metodología}

El proceso investigativo combinó procedimientos y enfoques cuantitativos y, principalmente, cualitativos, sobre todo por las continuas aproximaciones etnográficas que se realizaron a 104 comunidades de tres provincias de la Sierra Central (Cotopaxi, Tungurahua y Chimborazo). Tales aproximaciones etnográficas en las comunidades estudiadas fueron de permanencias cortas; es decir, el equipo de investigación visitaba y participaba de las actividades comunitarias por un día; durante la visita el trabajo consistía en identificar a las personas que puedan facilitar el acceso al grupo (Goetz y LeCompte, 1988). Habitualmente este acceso y el diálogo con los comuneros se produjo gracias a las convocatorias realizadas por los presidentes o síndicos de las mismas localidades. Esta particularidad de aproximación a los comuneros da cuenta de la estructura jerarquizada y vertical que aún perdura en la zona andina; por ello, es difícil realizar un acercamiento a las comunidades sin pasar por el filtro de la dirigencia comunitaria.

Una vez identificados los actores comunitarios se procedió a entablar un diálogo en el que los tópicos de la conversación giraban alrededor de la vida comunitaria y las problemáticas que se producían en ella. Este diálogo no contenía un guión de preguntas; por el contrario, correspondía a un listado de temas que le interesaba abordar al equipo de investigación.

Otra técnica que se empleó para la captura de la información y datos fue el relato de vida, técnica que permitió co-producir información debido a las cons-

Levantamiento o agitación 
tataciones ${ }^{1}$ que se hicieron durante la conversación. Finalmente, se debe mencionar que las conversaciones tematizadas (Goetz y LeCompte, 1988), en un primer momento, se realizaron de forma focalizada y diferenciada por rango etario; esta decisión de trabajar por grupos de edad obedeció a la lógica comunal andina, en donde el viejo o adulto impone su voz y discurso.

Sin embargo, a medida que el trabajo se realizaba se decidió modificar la metodología, planteando diálogos de forma heterogénea e integral sin distinción etaria; esto evidenció que los jóvenes también presentan argumentos y puntos de vista ahora validados y reconocidos por los adultos frente a los ámbitos planteados en la conversación.

\section{Resultados}

\section{Transformaciones de la educación indígena en la Sierra Central}

Si bien el paso del tiempo ha definido transiciones y cambios en la relación entre las instituciones educativas y el mundo indígena, debemos mencionar que también existen continuidades o permanencias. Una de ellas, y quizá la más indudable, responde a la matriz colonizadora (Unda y Muñoz, 2011) que determina asimetrías y rasgos de distinción (Bourdieu, 1996) entre los sujetos que son parte del sistema educativo y de aquellos que se encuentran al margen del mismo sistema escolarizado.

En el mundo indígena, y de forma particular en las provincias de Cotopaxi, Tungurahua y Chimborazo, ubicadas en la sierra del centro de Ecuador, la relación y la vinculación entre la escuela y comuneros se ha producido por intervenciones de sectores pertenecientes a la iglesia; es decir, el ingreso de misiones religiosas a las comunidades permitió que varios comuneros accedieran al sistema educativo y acumularan además de representación comunal y clerical un capital escolar que los sitúa como diferentes e incluso "superiores" al resto de sus comuneros.

Las marcadas diferencias se evidencian con mayor facilidad en los actuales momentos, debido a una constante participación de jóvenes indígenas cuyos niveles de instrucción y de escolarización son superiores a los logros obtenidos por sus antecesores llegando a convertirse en el segmento de dirigencia y representativo de sus comunidades. Es claro y casi obvio, que en el marco

1 Las constataciones consistían sobre todo en verificar o negar la información secundaria previamente revisada acerca de la comunidad visitada. 
de un proyecto cultural que se presentó desde la modernidad, la razón como principio y herramienta sea lo fundamental para construir una nueva forma de pensamiento y organización social (Dávila, Ghiardo y Medrano, 2008) y es justamente, sobre la base de esta razón ilustrada que las actuales generaciones de niños y jóvenes indígenas plantean una nueva forma de organización comunitaria, provocando una ruptura entre lo que significa y significaba ser un dirigente comunitario; es decir:

"Para estar al frente de una comunidad es necesario pasar por la escuela y saber qué decir a los demás" (Dirigente de la comunidad de Achupallas, cantón Alausí, provincia de Chimborazo).

En definitiva, la apuesta por la educación y por ser parte del sistema escolarizado se ha convertido en el impulso necesario para que el miembro de una comunidad sea reconocido y nombrado como representante y actor social de ese determinado contexto.

Pero el impulso educativo no fue una constante en la vida comunitaria de los pueblos indígenas andinos; por el contrario, lo realmente sustancial era la participación en procesos de intifadas y levantamientos que pretendían cristalizar demandas planteadas por varias décadas. Una de las principales demandas fue el salir del modelo hacendatario ${ }^{1}$ planteado para ese entonces ${ }^{2}$, pero la pretensión del cambio de modelo y de relaciones y el paso de ser indios parte de la hacienda a ser indios libres y administradores de sus propias tierras trajo consigo nuevos retos para los comuneros. Al ser ellos los nuevos propietarios y administradores de las tierras eran los encargados de comercializar los productos que en esas parcelas se producían, provocando así una integración e incursión en el mundo del comercio que obligó a los adultos a replantearse el modelo de enseñanzaaprendizaje comunitario.

Dicho replanteamiento exige que las nuevas generaciones de niños y jóvenes sean parte de la tarea civilizatoria (García, 2000) que la escuela imparte, dando paso a otro cambio comunitario fundamental que es el uso del lenguaje. Es decir, a partir de la incursión en el sistema escolarizado los niños paulatinamente

1 Modelo basado en la explotación de la mano de obra indígena por parte de los propietarios de las haciendas; irrespetando los derechos fundamentales de los trabajadores (percibir un salario por sus actividades, horas de descanso, etc.).

Sólo hasta 1973 que se realiza la reforma agraria en el Ecuador los indígenas andinos logran ser asumidos como indios libres y dejan de ser tratados como parte del inventario de la hacienda, Ampliar en Udo Oberem (1979) y J. Sánchez Parga (2002) 
dejan de lado su lengua vernácula -kichua- para aprender un lenguaje -castellano- socializante e integrador que asegura el anexo y participación en el mundo mestizo, comercial y urbano.

En la misma línea de la vinculación con la urbe, durante varios años la presencia de profesores mestizos y casi en su totalidad provenientes de las cabeceras cantonales y de las urbes aledañas a las comunidades significó una transformación en el proceso de enseñanza-aprendizaje. Tales cambios operaron en dos sentidos. i) El sistema escolar comunitario asumió pautas pedagógicas provenientes de contextos urbanos, dejando de lado las prácticas culturales propias del aprendizaje comunitario, es decir, que el trabajo siempre se debía realizar de forma cooperativa y colaborativa, mientras que la pauta urbana supone la actividad escolar como una práctica individual e individualizante. ii) La instrucción y transmisión de conocimientos sólo se realizaba y -se realizaen castellano, lo que obligó a que los comuneros fuesen bilingües para poder ser parte del sistema escolar.

En el sistema escolar, este particular bilingüismo tiene sus repercusiones puesto que la lengua hablada y la escrita no son una misma expresada de dos formas diferentes, sino que son dos distintas (Sánchez, 2009, p. 73); esto supone, en palabras de Louis Not (1974) que la diferencia también es intelectual, ya que no se trata de una misma realidad mental expresándose por dos medios diferentes, sino de dos realidades mentales diferentes. En suma, la imposición lingüística en el espacio escolar significa una separación de sus estructuras y representaciones mentales aprendidas en su cotidianidad y que les obliga a representar un mundo, aún desconocido con una lengua que la están conociendo y aprendiendo.

\section{Comunidad, familia y escuela}

Las comunidades indígenas andinas se han caracterizado a lo largo de la historia por sortear diferentes obstáculos, dificultades que van desde los procesos de exclusión del cual han sido víctimas de las instituciones tanto públicas como privadas hasta la ubicación geográfica en la que se encuentran asentadas ${ }^{1}$. Pero a pesar de estas y otras vicisitudes la comunidad indígena andina ha podido

1 En la provincia de Chimborazo, sobre todo, existen comunidades que se encuentran asentadas a 4000 y más metros de altura sobre el nivel del mar. Por encontrarse en un piso climático de tal altitud, la producción agrícola es escasa, por lo tanto, los habitantes de comunidades como Cobshi y Ozogoche habitualmente se dedican a la crianza de animales de páramo como son ovejas y llamas. 
permanecer y sostenerse en el tiempo, gracias a un fuerte lazo comunitario que ha empezado a desatarse debido a los constantes flujos migratorios del campo a la ciudad y en años recientes la diáspora se ha prolongado a países vecinos. Las causas son numerosas, pero los hallazgos del estudio realizado dan cuenta que son tres los elementos sustantivos que provocan y detonan el proceso de descomunalización (Sánchez, 2002) en el mundo andino.

\section{Procesos migratorios}

Aunque el fenómeno migratorio rural- urbano no es nuevo (Unda, 2010, p. 47) se debe mencionar que los flujos migratorios tienden a amplificarse e intensificarse a partir de los procesos de modernización del estado ecuatoriano -1970. Sin embargo, el paso de un modelo de economía primaria de agroexportación a una economía primaria fundamentalmente extractiva, basada en la explotación de petróleo (Acosta, 1987) fue el impulso fundamental para que los flujos migratorios del campo a la ciudad crezcan notablemente.

Con el antecedente antes mencionado podemos decir, que la constante incursión de indígenas a la ciudad respondió sobre todo a la búsqueda de plazas laborales, es decir, la demanda de trabajo se convirtió en el punto central para que el hombre migre de su comunidad a los polos económicos en desarrollo -Quito, Guayaquil y más tarde Cuenca-.

Pero la búsqueda de plazas laborales no fue la única razón por la que la diáspora indígena se intensificó; por el contrario, con la incorporación de comuneros provenientes de la zona andina al mundo del trabajo aparecen razones vinculadas a la certificación que avalen el trabajo a realizar. En ese sentido, los trabajadores indígenas se vieron obligados a incursionar en el proceso educativo, convirtiendo la escuela en la institución acreditadora y reconocedora de sujetos calificados para el mundo laboral urbano. Sin embargo, a pesar de la creación de escuelas en zonas rurales, las familias optaron -y se mantiene la preferencia-, porque sus hijos se inserten en centros escolares de las cabeceras cantonales y ciudades más cercanas. Esta decisión responde al imaginario colectivo comunitario de creer que la educación urbana es de mejor calidad y que con esta incursión en centros escolares alejados de su contexto comunitario, se asegura un puesto laboral mejor remunerado.

En síntesis, la relación entre vinculación laboral y escuela han sido los principales factores que motivaron el éxodo del campo a la urbe. 


\section{La intensificación del uso y consumo de Tecnología de Información y comunicación TIC}

Con la llegada de la primera década del siglo XXI, el acceso, uso y consumo de las tecnologías de la información y comunicación se acrecentó, y sus ventajas cubrieron las zonas rurales andinas del Ecuador ampliando así las expectativas de los comuneros y de forma particular en los sectores poblacionales jóvenes. Por supuesto, el uso y consumo de las TICs se encuentra asociado al proceso migratorio, pues son los comuneros que han tenido una aproximación con las zonas urbanas los que introducen las nuevas tecnologías a sus comunidades. Las nuevas tecnologías, según los testimonios recogidos a lo largo del estudio ${ }^{1}$, han modificado y transformado las relaciones comunales a todo nivel provocando una "fractura comunicativa", algo que en un principio puede ser asumido como una paradoja pero que en la cotidianidad comunal está sucediendo sobre todo porque los más jóvenes de las comunidades mantienen una lejanía con las prácticas comunales:

"Los jóvenes ya no participan de las actividades comunitarias, como las mingas y asambleas porque a ellos sólo les interesa estar con el celular, escuchando música y ahora último pasan en el internet" (entrevista a comunero de Pasa San Fernando en la provincia de Tungurahua)

Como se puede apreciar en este testimonio, la percepción de los adultos es referida a la ausencia de participación comunal, lo que ha provocado una reconfiguración comunitaria, ya que son los adultos mayores, hombres, mujeres e incluso niños los que mayoritariamente colaboran en actividades colectivas que siempre fueron patrimonio comunal. Esta referida descomunalización de las actividades productivas es el resultado de una creciente privatización e individualización de las estrategias económicas del mercado (Sánchez, 2009, p. 40) que son ampliamente difundidas y expandidas por las Tics.

\section{Ingreso de iglesias y agencias de cooperación en el mundo indígena}

A partir de 1960, los procesos de evangelización en América Latina y en el Ecuador se intensificaron y, por supuesto, el mundo indígena no quedó al margen de esta campaña religiosa, a tal punto que la cristianización, la catequesis y las jornadas de alfabetización llegaron a espacios geográficos que la mano estatal no había logrado penetrar. En ese sentido, el contacto educativo que tuvieron

1 Cuando en el trabajo de campo se conversó con los cabezas de familia y dirigentes comunitarios existió una respuesta común y era referida a la ausencia de vida comunitaria por parte de los jóvenes, para ellos. los adultos- el uso de medios comunicativos es un agente distractor y disociador de la vida comunitaria 
los pobladores de comunidades indígenas andinas, fue mediado por los valores cristiano-católicos y cristiano -evangélicos.

Pero la doctrina cristiana no fue la única que ingresó a los poblados indígenas. Con la promulgación de la primera reforma agraria en el Ecuador (1964) llegó el contingente de Alianza para el Progreso, que constituyó un frente de colaboración y ayuda a los comuneros que habían sido beneficiados con la entrega de tierras. Las principales estrategias de colaboración consistían en instalar espacios de capacitación que contribuyeran al manejo de las tierras adjudicadas y animar capacidades técnicas que necesariamente debían estar bajo acompañamiento de procesos educativos. Es por ello que Alianza para el Progreso inyecta recursos económicos favoreciendo la instalación de centros educativos en zonas rurales con predominancia indígena.

A medida que los años transcurren, la población indígena empieza a convertirse en agente de beneficios y los donantes cooperantes se diversificaron. Es así que se consolidó el ingreso de una amplia cartera de agencias de cooperación, las mismas que se encargaron de consolidar los procesos de capacitación técnica en el manejo de tierras y animales, alfabetización y capacitación en temas vinculados a los derechos de género, infancia y salud reproductiva. Todo este ingreso de recursos de la cooperación movilizó a un amplio sector de comuneros, los mismos que empezaron a capacitarse, para luego ser ellos los agentes multiplicadores de lo aprendido. Pero claro, con la capacitación y la formación adquirida, los procesos de distinción entre los miembros de la comunidad se amplían, pues a partir de este proceso de formación aparece un segmento de comuneros que son los que ahora dirigen los destinos de la comunidad. En suma, la idea fundamental que las ONGs tienen a propósito de capacitar y formar para igualar y mejorar las condiciones de los habitantes de las comunidades, tiene un resultado inverso, pues el tejido social y el valor por el trabajo colectivo basado en la autoridad de los ancianos se transforma y la autoridad ahora la ostenta el que sabe y conoce, y todo aquel que cuente con las herramientas tecnológicas que amplían y profundizan el conocimiento.

\section{La escuela y el mundo laboral}

En sintonía con lo expuesto líneas arriba, se puede decir que la relación escuela y mundo laboral se ha convertido en otro puntal que afianza las asimetrías entre los comuneros de la Sierra Central ecuatoriana. Las razones son múltiples, pero quizá la más significativa circula alrededor de lo que los mismos miembros de las comunidades aseguran:

Para $\mid$ Llanos Erazo, Daniel (2013). Asimetrías y aproximaciones a la problemática educativa de 
"Todos los niños terminan la escuela, el colegio tienen que estudiar afuera de la comunidad, porque aquí no hay, pero claro, los que terminan el colegio son los que consiguen mejores trabajos, casi siempre en las ciudades (...) ellos cuando regresan vienen con cosas y ayudan a sus familias" (madre de familia de la comunidad de Guargualla Grande).

Claramente se puede apreciar que la posibilidad de acceder al sistema educativo demarca el camino a la inserción laboral y, por supuesto, a un trabajo que asegure y posibilite una adecuada cantidad de ingresos que contribuya al mejoramiento de la calidad de vida. Es así, que la mayoría de comuneros adultos consideran que el mayor acceso a la educación asegura un progreso económico y claramente una mejoría en las condiciones de vida de las familias, aunque esto signifique un cambio cultural para todo el núcleo filial y la comunidad debido a la importación y adopción de modas y consumos culturales ajenos a sus tradiciones y costumbres vernáculas. Dichas modas se evidencian desde la estética corporal (piercings, tatuajes, aretes) hasta el diseño y construcción de las nuevas viviendas. Los modelos arquitectónicos de las casas ahora cuentan con estilos, materiales y detalles urbanos, elementos que refieren a los propietarios como sujetos que trabajan fuera y han logrado un nivel de escolaridad superior al resto de comuneros.

Otros elementos que intervienen en la relación educación, trabajo y proyección laboral son los sistemas de imaginarios y expectativas, los mismos que responden a lógicas de matriz urbanizadas, es decir, los comuneros jóvenes y en su mayoría los adultos, descartan la posibilidad de que las nuevas generaciones se queden trabajando en el agro y la ganadería. Los nuevos horizontes laborales se plantean alrededor de adquirir una capacitación técnica, o una profesionalización intermedia, -técnicos superiores, tecnólogos ${ }^{1}$-, está formación, de acuerdo con los testimonios recogidos, asegura una promoción laboral fuera de sus terruños, lo que conlleva a que los migrantes capacitados y estudiados sean considerados como los "héroes" de la comunidad, pues han alcanzado el tan anhelado y buscado progreso.

Finalmente, la relación entre escuela y mundo laboral presenta la variable de género como un elemento sustantivo, al interior de las familias, a la hora de la decisión del miembro que estudiará, a saber que las familias y la misma comunidad indígena auspiciaban e incluso autorizaban para que sean los hombres quienes

Aunque existen comuneros que consideran que la capacitación técnica y profesionalización intermedia puede contribuir al mejoramiento de las condiciones agraria y pecuarias de las comunidades, también existen quienes consideran que el estudio necesariamente les obliga a buscar espacios laborales fuera de su sector y por supuesto, la idea de migrar dentro o fuera del país es constante. 
estudien, dejando de lado a las mujeres. Este tipo de comportamientos, obedecía a la herencia de la matriz machista, en la cual la mujer era confinada al cuidado del espacio doméstico negando la posibilidad de incursionar en actividades que se aseguraban era de exclusiva dedicación masculina. Pero estas prácticas se han transformado en los últimos veinte años por la masiva incursión de mujeres dentro del sistema escolar, promoviendo un considerable contingente de mujeres que son participes de actividades laborales que históricamente les fueron negadas ${ }^{1}$.

\section{Conclusiones}

El ingreso de las agencias de cooperación al igual que las misiones religiosas y carismáticas determinó una transformación en el mundo comunitario indígena de la Sierra Central ecuatoriana debido, sobre todo, al proceso de evangelización y catequesis que se impartieron en el sistema escolar. Es decir, las primeras escuelas que se instalaron en la zona andina no fueron únicamente motor de instrucción, por el contrario, parte fundamental de su quehacer educativo fue el impartir valores religiosos y sociales que se oponían a los principios comunales.

En ese mismo sentido, los valores que la escuela irradiaba en las comunidades iban estableciendo nuevos patrones de comportamiento, los mismos que provocaron un giro en las visiones y relaciones familiares como comunales, a saber, que estos dos espacios se encuentran íntimamente ligados a la producción social y económica de las localidades los efectos fueron inmediatos, a tal punto que la idea de migrar a las urbes más cercanas para alcanzar el anhelado progreso y desarrollo se convirtió en una constante en los últimos 50 años y que, en la última década la diáspora se intensificó, a tal punto que en las comunidades la presencia de jóvenes es cada vez menor dejando así un espacio "comunitario" formado principalmente por mujeres, niños y ancianos.

Finalmente, se puede decir que la estrecha relación existente entre sistema escolar y mundo laboral determina una nueva dinámica socio comunitaria (mixturizada y casi urbanizada), ante todo por los constantes flujos que se producen a partir de estas dos actividades que necesariamente obligan a los comuneros a trasladarse a urbes próximas provocando tensiones culturales entre adultos y jóvenes.

Un ejemplo a citar es la actual Prefecta de la provincia de Cotopaxi, Blanca Guamangate quien cuenta con una licenciatura en educación y ha venido ocupando diferentes cargos políticos en su provincia. 


\section{Referencias}

Acosta, A. (1987). La lógica del capital financiero internacional. Quito: CNE.

Bourdieu, P. y Passeron, J. C. (1996). La Reproducción. Elementos para una teoría del sistema de enseñanza. México: Ed. Fontmara.

Bourdieu, P. y Passeron, J. C. (1996). Cuestiones de sociología. Madrid: Istmo.

Cerbino, M. (2000). Culturas juveniles, cuerpo, música, sociabilidad y género. Ecuador: Abya Yala. Editorial.

Cerbino, M. (2004). Pandillas juveniles, cultura y conflicto de la calle. Ecuador: Abya Yala. Editorial.

Cerbino, M. (2008). Otras naciones, jóvenes, transnacionalismo y exclusión. Ecuador: FLACSO y Ministerio de Cultura.

Dávila, O., Ghirardo, F., y Medrano, C. (2008). Los Desheredados: Trayectorias de vida y nuevas condiciones juveniles. Santiago de Chile:

García, N. (2000). La globalización imaginada. México: Paidós.

Goetz, J.P. y Lecompte, M. D. (1988). Etnografía y diseño cualitativo en investigación educativa. Madrid: Ediciones Morata.

Not, L. (1997). Les pedagogies de la connaissance. Toulousse: Ed. Privat.

Obeerem, U. (1979): Indios libres e indios sujetos a haciendas en la Sierra Central ecuatoriana a fines de la colonia. En I,Haus Volker und Kulturen Amerikanische Studien. St. Augustin.

Pérez, J. A. (2008). Teorías sobre la Juventud. Las miradas de los clásicos. México: Ed. Porrúa.

Sánchez, J. (2007). El movimiento indígena ecuatoriano. Quito: CAAP.

Sánchez, J. (2002). Crisis en torno al Quilotoa. Mujer, cultura y comunidad. Quito: CAAP.

Sánchez, J. (1993). Transformaciones socioculturales y educación indigena. Quito: CAAP.

Sánchez, J. (1989). Faccionalismo, organización y proyecto étnico en Los Andes. Quito: CAAP.

Sánchez, J. (1984). La trama del poder en la comunidad andina. Quito: CAAP.

94 Sánchez, J. (2009) Qué significa ser indígena para el indigena. Quito: Abya yala.

Para 
Stephen, J. (Comp.). (1994). Foucaulty la educación, disciplina del saber. Madrid: Morata.

Unda, R. (2010). Jóvenes y juventudes. Acción, representaciones y expectativas sociales de jóvenes en Quito. Quito: Abya Yala.

Unda, R., y Muñoz, G. (2011). La condición juvenil indígena. Elementos iniciales para su construcción conceptual. Última Década 34.

Para $\quad$ Llanos Erazo, Daniel (2013). Asimetrías y aproximaciones a la problemática educativa de Autónoma de Manizales. ISSN 0121-6538. 